\title{
Perfis antropométrico, lipídico e glicêmico em adolescentes de uma instituição filantrópica no noroeste do Paraná
}

Primeira submissão em 28/07/09 Última submissão em 13/01/10 Aceito para publicação em 18/01/10 Publicado em 20/02/10

\author{
Anthropometric, lipids and blood glucose profiles in adolescents of a \\ philanthropic institution in the Northwest Paraná
}

Anderson da Silva Honorato'; Érika Bando²; Taqueco Teruya Uchimura³; Miguel Machinski Junior ${ }^{4}$

unitermos
Adolescentes
Perfil antropométrico
Perfil lipídico
Índice de massa corporal

Indice de massa corporal

\section{resumo}

Introdução: A obesidade na adolescência é um fator preditivo da obesidade no adulto. A prevalência em crianças e adolescentes tem aumentado na maior parte dos países e tem-se traduzido em um dos mais significativos problemas nutricionais da atualidade. Objetivos: Este estudo teve por objetivo verificar os perfis antropométrico, lipídico e glicêmico em adolescentes de baixo nível socioeconômico de uma instituição filantrópica no noroeste do Paraná. Material e métodos: A população estudada foi composta por 92 adolescentes, com idade entre 10 e 17 anos. Foi utilizado o índice de massa corporal (IMC) para verificar o estado nutricional dos adolescentes, considerando normal $24,9 \mathrm{~kg} / \mathrm{m}^{2}$ e sobrepeso > $25 \mathrm{~kg} / \mathrm{m}^{2}$. A razão cintura-quadril (RCQ) foi mensurada por meio do quociente da cintura pelo quadril, e as concentrações de glicose, triglicerídeos, colesterol total, frações HDL-C, LDL-C e VLDL-C foram determinadas segundo o método enzimático colorimétrico. Resultados: Encontraram-se 9,79\% de sobrepeso nos adolescentes e $9,78 \%$ de RCQ e colesterol inadequados para a idade. Baixa correlação foi encontrada entre IMC e sobrepeso, glicemia, hipercolesterolemia, hipertrigliceridemia, LDL-C, VLDL-C e HDL-C, enquanto idade (razão de chance $[R C]=49,16)$ e $R C Q(R C=13,99)$ apresentaram correlação significativa $(p<0,05)$ com IMC. Conclusão: $O$ resultado demonstra a necessidade de políticas públicas de saúde no âmbito escolar como fator preventivo da obesidade e seus fatores de risco.

\section{abstract}

Introduction: Obesity in adolescence is a predictive factor of obesity in adulthood. The prevalence in children and adolescents has increased in most countries and has become one of the most significant nutritional problems nowadays. Objectives: This study aimed at verifying the anthropometric, lipid and glycemic profiles in lower class adolescents from a philanthropic institution located in the Northeast of Paraná State. Material and method: The studied population consisted of 92 adolescents, from 10 to 17 years of age. The Body Mass Index (BMI) was used to verify their nutritional status, considering $\leq 24.9 \mathrm{~kg} / \mathrm{m}^{2}$ normal and > $25 \mathrm{~kg} / \mathrm{m}^{2}$ overweight. The waist-to-hip measurement was calculated through waist-to-hip ratio (WHR) and glucose concentrations, triglycerides, total cholesterol, HDL-C, LDL-c and VLDL-c fractions were determined through enzymatic methods. Results: $9.79 \%$ were overweight and $9.78 \%$ of WHR and cholesterol levels were inadequate to the age group. BMI (overweight), blood glucose, hypercholesterolemia, hypertriglyceridemia, $L D L-C, V L D L-c$ and HDL-c showed low correlation, whereas age $(O R=49.16)$ and WHR $(O R=13.99)$ showed significant correlation $p<0.05$. Conclusion: The result evidenced the necessity of health public policies in educational scope in order to prevent obesity and its risks factors in adolescence.

\section{key words}

Adolescents

Anthropometric profile

Lipids profile

Body mass index

\footnotetext{
1. Mestrando do Curso de Pós-Graduação em Ciências da Saúde da Universidade Estadual de Maringá (UEM).

2. Farmacêutica-bioquímica do Laboratório de Toxicologia da UEM.

3. Professora associada do Departamento de Enfermagem da UEM.

4. Professor associado da disciplina de Toxicologia do Departamento de Ciências Básicas da Saúde da UEM.
} 


\section{Introdução}

A obesidade é considerada pela Organização Mundial da Saúde (OMS) um importante problema mundial de saúde pública, pois está associada a hipertensão arterial, doença cardíaca, osteoartrite, diabetes tipo II e alguns tipos de cânceres, tendo impacto mais pronunciado na morbidade do que na mortalidade $(7,48,50)$. A prevalência em crianças e adolescentes tem aumentado na maior parte dos países e tem-se traduzido em um dos mais significativos problemas nutricionais da atualidade ${ }^{(29,49)}$.

Cronologicamente, a adolescência corresponde ao período de 10 a 19 anos, sendo esse período dividido em duas fases: 10 a 14 anos e 15 a 19 anos. A faixa etária compreendida entre 10 e 14 anos inclui o início das mudanças puberais. O término da fase de crescimento e o desenvolvimento morfológico ocorrem no período que corresponde à segunda fase $\mathrm{e}^{(51)}$.

Essa patologia tem se tornado frequente mesmo em nações em desenvolvimento, nas quais persistem regiões e grupos sociais submetidos a contextos de fome e desnutrição. Estudos apontam, inclusive, para a concomitância de casos de desnutrição e de obesidade na mesma moradia ${ }^{(6,22)}$.

Quanto mais prevalente se torna a obesidade, maior o estímulo para estudar grupos populacionais mais vulneráveis ao problema, em que se possam levantar hipóteses relacionadas à determinação dessa patologia. Intervenções na infância e na adolescência são importantes, pois são períodos críticos para o desenvolvimento da obesidade. Assim, são recomendadas implementação de atividades preventivas de problemas de saúde e nutrição específicas dentro das políticas de saúde pública como forma de evitar os desfechos desfavoráveis na idade adulta ${ }^{(15,24,26)}$.

A OMS vem enfatizando a necessidade da realização de estudos sobre níveis de crescimento em populações pertencentes a países subdesenvolvidos e/ou desenvolvidos ${ }^{(21)}$, uma vez que essas informações auxiliam no atendimento $\mathrm{e}$ no acompanhamento de programas voltados à promoção da saúde dessas populações. Para que haja intervenções efetivas, torna-se necessário o desenvolvimento de avaliações periódicas por intermédio de medidas antropométricas e avaliações nutricionais que procuram fornecer informações quanto aos parâmetros de crescimento de adolescentes.

Os fatores associados à ocorrência da obesidade têm sido identificados entre as variáveis sociais, ambientais, biológicas e econômicas ${ }^{(36,38)}$. A relação entre o nível socioeconômico e a obesidade infantil não tem sido consistente na literatura científica, entretanto, uma relação inversa, de redução de obesidade com o aumento da renda familiar, tem sido demonstrada em alguns estudos ${ }^{(8,16)}$.

A prática de atividade física diminui o risco de aterosclerose e suas consequências, como angina, infarto do miocárdio e acidente vascular cerebral, ajuda no controle de obesidade, hipertensão arterial, diabetes, osteoporose e dislipidemias e diminui o risco de afecções osteomusculares e de alguns tipos de cânceres (colo e mama). Contribui ainda no controle de ansiedade, depressão, doença pulmonar obstrutiva crônica e asma, além de proporcionar melhora na autoestima e de ajudar no bem-estar e na socialização do indivíduo ${ }^{(3,9)}$.

Sabe-se que o peso corporal e a quantidade de gordura corporal são os principais moduladores dos níveis de pressão arterial e de lipídios plasmáticos em crianças e adolescentes. Quando se apresentam proporções de triglicerídeos (TCL), colesterol total (CT), colesterol da lipoproteína de baixa densidade (LDL-C) e colesterol da lipoproteína de muita baixa densidade (VLDL-C) acima dos limites desejáveis para a idade, associadas à diminuição nos valores de colesterol da lipoproteína de alta densidade (HDL-C), aumenta a probabilidade do desenvolvimento de doenças cardiovasculares ${ }^{(41,46)}$.

O início da aterosclerose já na infância, pelo aumento do colesterol plasmático, seria potencializado no decorrer da vida pela obesidade e por uma série de outros fatores, tais como história familiar, inatividade física e hipertensão arterial(35), sugerindo a necessidade de estratégias para prevenção dos fatores de risco, ainda na fase infanto-juvenil(31).

Um aumento na quantidade de gordura visceral é considerado um dos mais relevantes fatores de risco para o desenvolvimento de doenças cardiovasculares, diabetes e outros problemas metabólicos. Há vários métodos para avaliar a gordura visceral; um dos mais simples, de fácil execução e reprodutibilidade é a relação entre as medidas da cintura e do quadril(2, 17).

\section{Objetivos}

Diante do exposto, este estudo tem por objetivo verificar o perfil antropométrico, lipídico e glicêmico em adolescentes de baixo nível socioeconômico de uma instituição filantrópica no noroeste do Paraná. 


\section{Material e métodos}

Trata-se de um estudo transversal descritivo-analítico, realizado em uma Instituição Filantrópica no Noroeste do Paraná no ano de 2006.

O Centro Social estudado possui caráter social com fins filantrópicos, tendo como mantenedora a Associação Brasileira de Ensino e Cultura (ABEC). Para participar do processo de seleção é necessário ter renda familiar per capita de, no máximo, meio salário mínimo, o que atende à Lei Orgânica de Assistência Social de 1993 (LOAS) ${ }^{(4)}$, tendo como prioridade os cadastrados em programas assistenciais do governo federal e matriculados em escola regular. $O$ estudo foi desenvolvido nessa Instituição devido ao grande número de adolescentes de baixo estrato econômico atendidos no município de Maringá-PR.

Do total de 490 crianças e adolescentes de 7 a 17 anos, foram selecionadas para a amostra $92(18,80 \%)$ adolescentes, porque estudavam no período vespertino e teriam condições de estar em jejum no período da manhã para a coleta de sangue, excluindo aqueles de outros períodos e as meninas que porventura estivessem gestantes no momento da coleta.

Os adolescentes foram divididos em dois grupos, conforme classificação etária proposta pela OMS: 10 a 14 anos e 15 a 17 anos $^{(51)}$. As medidas antropométricas (peso, estatura, circunferências de cintura e quadril e espessura da dobra cutânea tricipital e subescapular) foram realizadas em duplicata, sendo utilizadas a média aritmética dos valores, exceto as dobras cutâneas, que foram realizadas em triplicata.

A estatura foi medida por um estadiômetro de madeira, com escala de precisão de $0,1 \mathrm{~cm}$ com um cursor acoplado para facilitar a leitura. Cada adolescente foi colocado em pé e descalço sobre a base do estadiômetro com os pés unidos e membros superiores pendentes ao longo do corpo. Para saber o peso dos adolescentes, utilizou-se uma balança tipo plataforma (Filizola ${ }^{\circledR}$ com capacidade para até $150 \mathrm{~kg} \mathrm{e}$ precisão de $100 \mathrm{~g}$, situada em superfície plana.

A partir dos dados de peso e estatura, foi calculado o IMC, utilizando a equação de Quetelet, quociente do peso (em quilos) pelo quadrado da estatura (em metros): IMC = peso corporal $(\mathrm{kg}) /$ estatura $^{2}(\mathrm{~m})$. Adotaram-se os critérios de classificação de pontos de corte propostos pela $\mathrm{OMS}^{(50)}$, que correspondem ao programa utilizado para avaliação física proposto por Guedes e Guedes (1998), denominado Sistema de Avaliação e Prescrição da Atividade Física (SAPAF-
Jovem), que classifica IMC $\leq 24,9 \mathrm{~kg} / \mathrm{m}^{2}$ como normal e IMC $>25 \mathrm{~kg} / \mathrm{m}^{2}$ como sobrepeso.

Para as medidas de circunferência de cintura e quadril e espessura das dobras cutâneas tricipital e subescapular, cada adolescente foi colocado em posição ortostática, sem nenhum tipo de vestimenta sobre a região a ser manuseada. Todas as medidas foram realizadas no hemicorpo direito do avaliado, exceto as circunferências de cintura abdominal e quadril, que foram coletados na porção de maior circunferência com fita métrica inextensível, graduada em milímetro, por um único avaliador. A definição de distribuição central da adiposidade baseou-se na razão cintura-quadril, que é um importante indicador de risco coronariano, obtida pelo quociente entre as circunferências da cintura e do quadril: $\mathrm{RCQ}=$ cintura $(\mathrm{cm}) /$ quadril $(\mathrm{cm})$, sendo considerado normal $\leq 0,90 \mathrm{~cm}$ para homens $\mathrm{e} \leq 0,80 \mathrm{~cm}$ para mulheres ${ }^{(20)}$.

As medidas de espessura das dobras cutâneas foram mensuradas por meio de um adipômetro (Cescorf ${ }^{\circledR}$ ), coletadas por apenas um avaliador. A dobra cutânea tricipital foi determinada paralelamente ao eixo longitudinal do braço direito, na face posterior. O ponto exato de reparo se localizou na distância média entre a borda súpero-lateral do acrômio e o olécrano. A dobra cutânea subescapular foi destacada obliquamente ao eixo longitudinal, seguindo a orientação dos arcos costais, dois centímetros abaixo do ângulo inferior da escápula.

Utilizou-se a somatória das dobras para verificar o percentual de gordura relativa, sendo considerado normal $13 \%$ a $17 \%$ de gordura para meninos de 10 a 17 anos, $17 \%$ a $23 \%$ para meninas de 10 a 15 anos e $23 \%$ a $27 \%$ de gordura relativa de 16 a 18 anos ${ }^{(19)}$. Essa diferença é predita, pois as meninas apresentam o início do processo maturacional antes dos meninos, sendo que na fase púbere as diferenças sexuais geradas pela maturação propiciam o aumento de acúmulo de gordura nos adipócitos pelas meninas e nos meninos o ganho de massa muscular ${ }^{(25,27)}$.

A flexibilidade foi medida por meio do teste de sentar e alcançar, em que o avaliado com as palmas das mãos para baixo e em contato com o flexômetro, denominado Banco de Wells, estende-se à frente ao longo da escala de medida, realizando o movimento de modo lento e sem solavancos. O resultado final equivale à maior distância alcançada (em centímetros) pelas pontas dos dedos de ambas as mãos e mantida por aproximadamente 2 segundos, em um total de três tentativas, considerando normal os valores de percentis $\geq$ P50 e alterado < P50, conforme escala de valores proposta por Guedes e Guedes ${ }^{(20)}$. 
Já a resistência muscular foi medida por meio do teste abdominal, realizado quando o avaliado, na posição inicial, é colocado em decúbito dorsal sobre um colchonete, quadris e joelhos flexionados, plantas dos pés voltadas para o solo, braços cruzados sobre a face anterior do tórax, com as palmas das mãos voltadas para este na altura dos ombros opostos. O adolescente, ao sinal do avaliador, tinha 60 segundos para realizar o maior número de flexões abdominais. Utilizaram-se valores de percentis conforme Guedes e Guedes ${ }^{(20)}$, sendo considerado normal $\geq$ P50 e alterado $<$ P50.

Para os exames laboratoriais foram coletados $10 \mathrm{ml}$ de sangue pelos técnicos do laboratório no Centro Social. A coleta das amostras foi realizada por punção venosa após jejum de 12 a 14 horas, acondicionadas e transportadas para - Laboratório de Toxicologia da Universidade Estadual de Maringá (UEM). Para a determinação de glicose, TGL, CT e HDL-C foi utilizado o método enzimático-colorimétrico, enquanto o LDL-C e o VLDL-C foram calculados pela fórmula de Friedewald(12).

Os valores de referência para TGL, CT, LDL-C, HDL-C e VLDL-C foram os adotados segundo as normas das III Diretrizes Brasileiras sobre Dislipidemias ${ }^{(41)}$, e para glicemia, as normas da American Diabetes Association(1).

Os dados foram arquivados no programa Excel e posteriormente analisados no programa SAPAF-Jovem, Epi-Info e Statistica 6.0. Foram realizadas frequências simples para as variáveis descritivas e análises univariadas e multivariadas para as categóricas, observando o nível de significância de $5 \%$ para todos os testes.

Esse estudo foi aprovado pelo Comitê de Ética em Pesquisa Envolvendo Seres Humanos, conforme Resolução 196/96 do Conselho Nacional de Pesquisa e Parecer $n^{\circ}$ 405/2005. Todos os adolescentes apresentaram o termo de consentimento livre e esclarecido assinado por seus pais ou responsáveis, que informava os objetivos do estudo, a forma como seriam realizadas as coletas de sangue, a garantia do sigilo sobre os resultados e, nos casos em que o adolescente apresentasse resultados alterados, os pais seriam orientados a procurar tratamento médico.

\section{Resultados}

Dos 92 adolescentes estudados, 41 (44,56\%) eram do sexo masculino e 51 (55,44\%) do sexo feminino. O número de sobrepesos encontrados entre os adolescentes foi 9
$(9,78 \%)$, sendo $5(5,43 \%)$ do sexo masculino e $4(4,35 \%)$ do sexo feminino.

Como mostra a Tabela 1, as variáveis idade e razão cintura-quadril apresentaram associação com o IMC com $x^{2}=11$ e 8,84, respectivamente, com diferença significativa entre suas categorias com risco relativo $(\mathrm{RR})=15,74$ e intervalo de confiança (IC) (95\%) de 2,06 a 120,27 para variável idade e $\mathrm{RR}=8,43$ e IC (95\%) de 1,87 a 37,99 para a variável RCQ, diferenças estatisticamente significativas ( $p$ $<0,05$ ) entre os adolescentes em sobrepeso e não obesos.

Na variável idade, observou-se que $8(25,81 \%)$ dos adolescentes de 15 a 17 anos $(n=31)$ apresentaram sobrepeso, enquanto apenas $1(1,64 \%)$ de 10 a 14 anos $(n=61)$ teve IMC superior a $25 \mathrm{~kg} / \mathrm{m}^{2}(p=0,0005)$. A RCQ acima de $0,8 \mathrm{~cm}$ para o sexo feminino e acima de $0,9 \mathrm{~cm}$ para o sexo masculino apresentou diferenças significativas ( $p=$ 0,0022 ) entre adolescentes em sobrepeso e não obesos, pois $9(9,78 \%)$ dos sobrepesos apresentaram resultados da RCQ acima dos valores de referência.

Para as variáveis força abdominal e flexibilidade, expressas com teste abdominal e teste de sentar e alcançar, e gordura relativa não se observaram associação e diferenças significativas entre os adolescentes com sobrepeso e os não obesos.

Quanto aos parâmetros bioquímicos dos adolescentes avaliados, não foi observada associação significativa com o IMC, não tendo diferenças significativas estatisticamente. Os adolescentes avaliados que apresentaram sobrepeso não mostraram prevalência de hipercolesterolemia, hipertrigliceridemia, níveis elevados de LDL-C ou VLDL-C e níveis baixos de HDL-C (Tabela 2). A variável glicemia não foi apresentada na tabela por não ter apresentado resultados alterados, 54 a $95 \mathrm{mg} / \mathrm{dl}$, conforme as normas da American Diabetes Association (1). Não houve nenhum caso associado a diabetes mellitus nos adolescentes estudados.

Foram realizadas análises multivariadas para verificar a relatividade do potencial preditivo das correlações realizadas. As variáveis TGL, VLDL-C, LDL-C e gordura relativa foram retiradas da análise por se apresentarem como variáveis de confusão para a análise final. A não associação entre as demais variáveis, provavelmente, se deve ao pequeno número da amostra ou porque não existem diferenças significativas entre os dois grupos estudados. As variáveis com associação estatisticamente significativa $(p<0,05)$ ao IMC foram idade e RCQ (Tabela 3).

Em relação à idade, a análise multivariada demonstrou que os adolescentes entre 15 e 17 anos apresentaram RC 
Análise univariada dos adolescentes (10 a 17 anos) de Maringá-PR segundo 0 estado nutricional (IMC) e as variáveis antropométricas (sexo, idade, teste abdominal, teste sentar e

Tabela 1 alcançar, gordura relativa e RCQ)

\begin{tabular}{|c|c|c|c|c|c|c|c|}
\hline \multicolumn{8}{|c|}{ Índice de massa corporal $\left(\mathrm{kg} / \mathrm{m}^{2}\right)$} \\
\hline & $\begin{array}{l}\text { Total } \\
n(\%)\end{array}$ & $\begin{array}{c}\leq 24,9 \\
n(\%)\end{array}$ & $\begin{array}{l}\geq 25 \\
n(\%)\end{array}$ & $\chi^{2}$ & RR & IC & $p^{*}$ \\
\hline Sexo & & & & 0,12 & & & \\
\hline Masculino(M) & $41(44,56)$ & $36(39,13)$ & $5(5,43)$ & & 1,75 & $0,45-5,42$ & 0,5051 \\
\hline Feminino $(F)$ & $51(55,44)$ & $47(51,09)$ & $4(4,35)$ & & 1 & & \\
\hline Idade & & & & 11 & & & \\
\hline 15-17 anos & $31(33,70)$ & $23(25,00)$ & $8(8,70)$ & & 15,74 & $2,06-20,27$ & 0,0005 \\
\hline 10-14 anos & $61(66,30)$ & $60(65,21)$ & $\begin{array}{c}1(1,09) \\
0\end{array}$ & & 1 & & \\
\hline \multicolumn{8}{|l|}{ Teste abdominal } \\
\hline Alterado & $46(50,00)$ & $41(44,57)$ & $5(5,43)$ & & 1,25 & $0,36-4,36$ & 1 \\
\hline Normal & $46(50,00)$ & $42(45,65)$ & $4(4,35)$ & & 1 & & \\
\hline $\begin{array}{l}\text { Teste sentar e } \\
\text { alcançar }\end{array}$ & & & 1,61 & & & & \\
\hline Alterado & $38(41,30)$ & $32(34,78)$ & $6(6,52)$ & & 2,84 & $0,76-10,66$ & 0,2038 \\
\hline Normal & $54(58,70)$ & $51(55,44)$ & $3(3,26)$ & & 1 & & \\
\hline Gordura relativa & & & & 2,36 & & & \\
\hline Alterado & $67(72,83)$ & $58(63,05)$ & $9(9,78)$ & & 0 & 0 & 0,1071 \\
\hline Normal & $25(27,17)$ & $25(27,17)$ & $0(0)$ & & 1 & & \\
\hline $\mathrm{RCQ}$ & & & & 8,84 & & & \\
\hline Alterado & $27(29,35)$ & $20(21,74)$ & $7(7,61)$ & & 8,43 & $1,87-37,99$ & 0,0022 \\
\hline Normal & $65(70,65)$ & $63(68,48)$ & $2(2,17)$ & & 1 & & \\
\hline
\end{tabular}

\section{Tabela 2}

\section{Análise univariada dos adolescentes (10 a 17 anos) de Maringá-PR segundo 0 estado} nutricional (IMC) e os resultados de lipídeos e lipoproteínas

\section{Índice de massa corporal $\left(\mathrm{kg} / \mathrm{m}^{2}\right)$}

$\begin{array}{ccc}\text { Total } & \leq 24,9 & \geq 25 \\ n(\%) & n(\%) & n(\%)\end{array}$

Triglicerídeos

$>130 \mathrm{mg} / \mathrm{dl}$

$$
6(6,52) \quad 4(4,35)
$$

$2(2,17)$

$\leq 130 \mathrm{mg} / \mathrm{dl}$
$79(85,87)$

$7(7,61)$ $\chi^{2}$ 1,68

Colesterol total

$\geq 170 \mathrm{mg} / \mathrm{dl}$

22(23,92)

$18(19,57)$

$4(4,35)$

$<170 \mathrm{mg} / \mathrm{dl}$

70(76,08)

$5(5,43)$

1,23

RR

4,1
1

$1,08-15,58$

0,1042

$\begin{array}{ccc}2,55 & 0,75-8,66 & 0,2097\end{array}$


Cont.

LDL-C

$\geq 110 \mathrm{mg} / \mathrm{dl}$

25(27,18)

$<110 \mathrm{mg} / \mathrm{dl}$
$21(22,83)$

$4(4,35)$

$5(5,43)$
0,69

$$
\begin{array}{ccc}
2,14 & 0,63-7,35 & 0,2477 \\
1 &
\end{array}
$$

0,17

$\begin{array}{ccc}3,71 & 0,66-20,95 & 0,2683 \\ 1 & & \end{array}$

0,06

HDL-C

$\begin{array}{ccc}3(3,26) & 2(2,17) & 1(1,09) \\ 89(96,74) & 81(88,04) & 8(8,70)\end{array}$

$<35 \mathrm{mg} / \mathrm{dl}$

29(31,52)

$27(29,35)$

$2(2,17)$

$\geq 35 \mathrm{mg} / \mathrm{dl}$

$63(68,48) \quad 56(60,87)$

$7(7,61)$

0,62

$0,14-2,81$

0,7142

IMC: índice de massa corporal; $\chi^{2}$ : qui-quadrado; RR: risco relativo; IC: intervalo de confiança 95\%; $p^{*}$ : nível de significância de 0,05.

Análise multivariada dos adolescentes ( 10 a 17 anos) de Maringá-PR segundo as variáveis

Tabela 3 antropométricas (sexo, idade, teste abdominal, teste sentar e alcançar, gordura relativa e RCQ)

Variáveis $\mathrm{RC}$

IC

Sexo

Masculino (M)

1,07

$0,27-4,11$

0,9215

Feminino (F)

1

Idade

15-17 anos

49,16

$2,49-967,48$

0,0103

10-14 anos

1

Teste abdominal

Alterado

1,94

$0,41-9,12$

0,4005

Normal

1

Gordura relativa

Alterado

1,35

$0,35-5,11$

0,653

Normal

1

RCQ

Alterado

13,99

$2,06-94,77$

0,0068

Normal

RCQ: razão cintura-quadril; RC: razão de chance; IC: intervalo de confiança 95\%; $p^{*}$ : nível de significância de 0,05.

estatisticamente significativa $(p=0,0103)$ de sobrepeso 49,16 vezes maior que o grupo de 10 a 14 anos. A variável RCQ demonstrou RC estatisticamente significativa ( $p=$ 0,0068 ) de 13,99 vezes maior para adolescentes com RCQ alterada, ou seja, valores superiores a $0,8 \mathrm{~cm}$ e $0,9 \mathrm{~cm}$ para meninos e meninas, respectivamente.

\section{Discussão}

O presente estudo utilizou o IMC como indicativo de sobrepeso e obesidade ${ }^{(48)}$, pois ele tem sido o indicador mais utilizado para a triagem de adiposidade em adolescentes e é 
indicado em estudos epidemiológicos por sua relativa facilidade de mensuração e correlação com gordura corporal|(23).

$\mathrm{Na}$ população estudada, nenhum caso de obesidade foi encontrado, enquanto para sobrepeso foram observados $9,79 \%$ dos casos $(n=9)$. A proporção de sobrepeso mostrou-se inferior à relatada em estudo realizado em São Paulo(13), que demonstrou sobrepeso instalado em $19,6 \%$ dos adolescentes de 10 a 14 anos de baixo nível socioeconômico. Os achados no presente estudo foram inferiores em relação às pesquisas realizadas na região Sul, que apresentaram $24,5 \%$ de sobrepeso e $9 \%$ de obesidade ${ }^{(32)}$ e $20,9 \%$ e $5 \%{ }^{(47)}$, respectivamente, e superiores ao estudo de Neutzling et al. ${ }^{(34)}$, que identificou $7,7 \%$ de sobrepeso e obesidade em adolescentes de 10 a 19 anos. Estudos realizados em Maringá- $\mathrm{PR}^{(37)}$ e Maracaí ${ }^{(42)}$ indicaram, respectivamente, $10 \%$ e $10,2 \%$ de sobrepeso para adolescentes de 15 a 17 anos, resultados com valores próximos aos encontrados pelo presente estudo. Esse resultado pode ser justificado pela população estudada fazer parte de um grupo de baixo estrato econômico e serem menos vulneráveis à obesidade, cuja prevalência tende a aumentar com o nível socioeconômico(32).

Observou-se que dos $9,79 \%$ dos casos de sobrepeso, $8,7 \%$ compreendiam adolescentes entre 15 e 17 anos e apenas 1,09\%, entre 10 e 14 anos. O grupo de adolescentes com sobrepeso estava inserido no mercado de trabalho e, dessa forma, não praticava as atividades físicas proporcionadas pelo Centro Social. Esses adolescentes participavam apenas dos cursos profissionalizantes, enquanto o grupo de $10 \mathrm{a}$ 14 anos estava inserido em atividades físicas como dança e esportes. É possível que o estímulo do Centro Social à prática de exercícios físicos tenha constituído o diferencial observado.

A definição de distribuição central da adiposidade baseou-se na razão cintura-quadril, que é um importante indicador de risco coronariano. Do total de adolescentes, 9,78\% $(n=9)$ dos casos apresentaram RCQ acima dos valores de corte estabelecidos ${ }^{(20)}$. Em estudo desenvolvido com crianças de 7 a 9 anos em Santa Catarina por Soar et al.(45), observou-se maior correlação positiva entre IMC e perímetro da cintura quando considerados indivíduos em sobrepeso e obesos, sendo essas correlações estatisticamente significativas $(p<0,01)$. Entretanto, o IMC e a RCQ apresentaram valores extremamente baixos de correlação.

O estudo realizado na Região Metropolitana de São Paulo com jovens de idade igual ou superior a 20 anos demonstrou que os indicadores da obesidade centralizada representados pela RCQ e a circunferência da cintura estavam associados de maneira diversa a fatores de risco socioeconômicos, comportamentais e bioquímicos e atuaram na etiologia dessa morbidade. Ambas estavam relacionadas com sexo, idade e grau de atividade física. $\mathrm{O}$ risco de obesidade centralizada aumentava com a idade e era maior nas mulheres ${ }^{(30)}$. A correlação significativa nesse estudo foi da RCQ e do IMC com $p<0,05$, enquanto Marelli et al. (28) encontraram uma correlação significativa da RCQ com o nível de triglicerídeos. Esses resultados confirmaram que, mesmo durante a infância, a obesidade grave estava relacionada a alterações lipídicas e que a adiposidade central estava associada à piora desse perfil( ${ }^{28,39)}$.

Em relação ao perfil lipídico, encontraram-se $6,52 \%$ de TGL, 23,92\% de CT, 27,18\% de LDL-C, 3,26\% de VLDL-C e $31,52 \%$ de HDL-C na população estudada com valores inadequados para a idade. Os valores médios obtidos no perfil lipídico dos adolescentes de baixa renda em MaringáPR eram semelhantes comparados a outros estudos ${ }^{(5,14,33)}$.

O perfil lipídico deste estudo apresentou valores menores em relação a outros. No estudo realizado no Instituto do Coração do Hospital das Clínicas da Universidade de São Paulo(11), foram analisados 180 adolescentes e a frequência de exames alterados foi de $42,7 \%$ para CT, 31,9\% para TGL, 38,3\% para LDL-C e 14,2\% para HDL-C. Em outro estudo em São Paulo(40), a prevalência encontrada foi de $27,5 \%$, 13\%, 19,3\% e 13,8\% para CT, TCL, LDL-C e HDL-C, respectivamente. Quando comparados com este estudo, o LDL-C e o HDL-C tiveram valores abaixo daqueles encontrados. No estudo realizado em Londrina- $\mathrm{PR}^{(45)}$, após avaliar 624 indivíduos de laboratório particular, de 3 a 19 anos, os resultados alterados foram de $13,1 \%$ no CT, $22,5 \%$ no TGL, $14 \%$ no LDL-C e 19,2\% no HDL-C. Também foram inferiores os resultados alterados para TGL e HDL-C avaliados em Maracaí ${ }^{(43)}$ e Niterói( ${ }^{(4)}$.

Os resultados alterados no perfil lipídico deste estudo foram superiores aos valores demonstrados em Itajaí-SC ${ }^{(18)}$ com crianças e adolescentes de 3 a 14 anos: 3,1\% para CT, 4,7\% para TGL, 6,6\% para LDL-C e 17,9\% para HDL-C. Esses valores estão acima dos esperados, inadequados para essa faixa etária. Também estavam acima dos valores encontrados no estudo realizado por Rego Filho et al.(37), na cidade de Maringá-PR, que avaliaram 57 adolescentes de 15 a 17 anos encontrando $7 \%$ da população com níveis de colesterol acima do desejável para a idade.

Essa constatação pode ser justificada por alguns fatores, como hábitos alimentares relacionados à característica socioeconômica da população estudada. Apesar de os estudos a respeito da influência do nível socioeconômico no perfil lipídico não serem consensuais, pesquisas mostraram médias inferiores de CT, TGL e das lipoproteínas para o grupo com condições financeiras baixa, ainda que não tenham significância estatística ${ }^{(10)}$. 


\section{Conclusão}

Os resultados demonstraram que a faixa etária de 15 a 17 anos apresentou maior risco de sobrepeso em relação à faixa etária de 10 a 14 anos, demonstrando a importância da prática de atividade física como fator preventivo de obesidade. Quanto à interação perfil lipídico e sobrepeso, destacou-se a alteração de triglicerídeos na população estudada. Esses resultados reforçam a necessidade de estudos sobre intervalos de referência de perfil lipídico nessa faixa etária na população brasileira, bem como a implementação de políticas de saúde pública no âmbito escolar.

\section{Referências}

1. AMERICAN DIABETES ASSOCIATION. Standards of medical care in diabetes. Diabetes Care, v. 28, suppl. 1, 2005.

2. BLACKETT, P. R.; KITTREDGE, D. Hyperlipidemia in children. South Med J, v. 86, n. 10, p. 1083-92, 1993.

3. BRASIL. Ministério da Saúde. DATASUS. Estatísticas vitais - Mortalidade e nascidos vivos. Disponível em: <http:// www. portal.saude.gov.br/saúde/>. Acesso em: 01 set. $2006 a$.

4. BRASIL. Ministério do Planejamento, Orçamento e Gestão. Lei Orgânica de Assistência Social (LOAS). Lei oo 8742 de 07/12/1993. Disponível em: <http://www.mp.gov. br>. Acesso em: 01 set. 2006b.

5. BROTONS, C. et al. World wide distribution of blood lipids and lipoproteins in childhood an adolescence: a review study. Atherosclerosis, v. 139, p. 1-9, 1998.

6. DOAK, C. M. et al. The dual burden households and nutrition transition paradox. Int J Obes Relat Metab Disord, v. 129, n. 1, p. 129-36, 2005.

7. ECKERSLEY, R.M. Losing the battle of the bulge: causes and consequences of increasing obesity. Med J Aust, v. 174, p. 590-2, 2001.

8. ENGESTRON, E. M.; ANJOS, L. A. Relação entre o estado nutricional materno e sobrepeso nas crianças brasileiras. Rev Saúde Pública, v. 30, n. 3, p. 233-9, 1996.

9. ERLICHMAN, J.; KERBEY, A. L.; JAMES, W. P. Physical activity and its impact on health outcomes. Paper 1: The impact of physical on cardiovascular disease an all-cause mortality: an historical perspective. Obes Rev, v. 3, p. 257-71, 2002.

10. FONTAL, M. R.; ESPIN, A. R. Hipercolesterolemia en la poblacion adolescente. Revista de la Faculdad de Mecidina, v. 23, n. 1, p. 1-7, 2000.

11. FORTI, N. et al. Fatores de risco para doença arterial coronariana em crianças e adolescentes filhas de coronariopatas jovens. Arq Bras Cardiol, v. 66, p. 11923, 1996.

12. FRIEDEWALD, W. T.; LEVY, R. I.; FREDRICKISON, D. $\mathrm{S}$. Estimation of the concentration of low-density lipoprotein cholesterol in plasma without use of preparative ultracentrifuge. Clin Chem, v. 18, p. 499502, 1972.

13. GARCIA, G. C. B.; GAMBARDELLA, A. M. D.; FRUTUOSO, M. F. P. Estado nutricional e consumo alimentar de adolescentes de um centro de juventude da cidade de São Paulo. Rev Nutr, v. 16, n. 1, p. 41-50, 2003.
14. GERBER, Z. R. S.; ZIELIWSKY, P. Fatores de risco de aterosclerose na infância. Um estudo epidemiológico. Arq Bras Cardiol, v. 69, n. 4, p. 231-36, 1997.

15. GILL, T. P. Key issues in the prevention of obesity. Br Med Bull, v. 53, p. 359-88, 1997

16. GIUGLIANO, R.; CARNEIRO, E. C. Fatores associados à obesidade em escolares. J Pediatr, v. 80, n. 1, p. 17-22, 2004.

17. GORAN, M. I.; BALL, G. D. C.; CRUZ, M. L. Obesity and risk of type 2 diabetes and cardiovascular disease in children and adolescents. J Clin Endocrinol Metab, v. 88, n. 4, p. 1417-27, 2003.

18. GRILLO, L. P. et al. Perfil lipídico e obesidade em escolares de baixa renda. Rev Bras Epidemiol, v. 8, n. 1, p. 7581, 2005.

19. GUEDES, D. P.; GUEDES, J. E. R. P. Controle do peso corporal, composição corporal, atividade física e nutrição. Londrina: Midiograf, 1998.

20. GUEDES, D. P.; GUEDES, J. E. R. P. Crescimento, composição corporal e desempenho motor em crianças e adolescentes. São Paulo: CLR Balieiro, 1997.

21. GUEDES, D. P. Crescimento, composição corporal e desempenho motor em crianças e adolescentes do município de Londrina (PR), Brasil. Tese. São Paulo: Universidade de São Paulo, 1994.

22. GUIMARÃES, L. V. et al. Fatores associados ao sobrepeso em escolares. Rev Nutr, v. 19, n. 1, p. 5-17, 2006.

23. HIMES, L. J.; DIETZ, W. H. Guidelines for overweight in adolescent preventive services: recomendations from a expert committee. Am J Clin Nutr, v. 59, p. 307-16, 1994.

24. MAGALHÃES, V. C.; AZEVEDO, G.; MENDONÇA, S. Prevalência e fatores associadas a sobrepeso e obesidade em adolescentes de 15 a 19 anos das regiões Nordeste e Sudeste do Brasil, 1996 a 1997. Cad Saúde Pública, v. 19, supl. 1, p. 5129-39, 2003.

25. MALINA, R. M.; BOUCHARD, C. Atividade física do atleta jovem: do crescimento à maturação. São Paulo: Roca, 2002.

26. MALINA, R. M.; BOUCHARD, C. Growth, maturation and physical activity. New York: Versa Press, 1991.

27. MARCONDES, E. Desenvolvimento da criança: desenvolvimento biológico: crescimento. Rio de Janeiro: Sociedade Brasileira de Pediatria, 1994.

28. MARELLI, G. et al. Relation of body fat distribution to blood 
lipid pattern in severe childhood obesity. Int J Obes Relat Metab Disord, v. 17, suppl. 2, p. 38-45, 1993.

29. MARINS, V. M. et al. Overweight and risk of overweight in school children in the city of Rio de Janeiro, Brazil: prevalence and characteristics. Ann Trop Pediatrics, v. 22, n. 2, p. 137-44, 2002.

30. MARTINS, I. S.; MARINHO, S. P. O potencial diagnóstico dos indicadores da obesidade centralizada. Rev Saúde Pública, v. 37, n. 6, p. 760-67, 2003.

31. McGILL, H. C. et al. Origin of atherosclerosis in childhood and adolescence. Am J Clin Nutr, v. 72, p. 1307-15, 2000.

32. MONTEIRO, P. O. A. et al. Diagnóstico de sobrepeso em adolescentes: estudo do desempenho de diferentes critérios para o índice de massa corporal. Rev Saúde Pública, v. 34, n. 5, p. 506-13, 2000.

33. MOURA, E. C. et al. Perfil lipídico em escolares de Campinas, SP, Brasil. Rev Saúde Pública, v. 34, n. 5, p. 499-505, 2000.

34. NEUTZLING, M. B. et al. Overweight and obesity in Brazilian adolescents. It J Obes Relat Metab Disord, v. 24, p. 869-74, 2000.

35. PELLANDA, L. C. et al. Ischemic heart disease: prevention should begin in childhood. Jornal de Pediatria, v. 78, n. 2, p. 91-96, 2002.

36. RAMOS, A. M. P.; BARROS FILHO, A. A. Prevalência da obesidade em adolescentes de Bragança Paulista e sua correlação com a obesidade do país. Arq Bras Endocrinol Metab, v. 47, p. 663-8, 2003.

37. REGO FILHO, E. A. et al. Avaliação nutricional de um grupo de adolescentes. Acta Sci Health Sci, v. 27, n. 1, p. 63-7, 2005.

38. RIBEIRO, I. C.; TADDEI, J. A. A. C.; COLUGNATTI, F. Obesity among children attending elementary public schools in São Paulo, Brazil: a case-control study. Public Health Nutr, v. 6, n. 7, p. 659-63, 2003.

39. ROLLAND-CACHERA, M. F.; DEHEEGER, M. Correlations between anthropometric indicators of abdominal fat and fatness indices in children. Int $J$ Obes Relat Metab Disord, v. 22, suppl. 4, p. 11-9, 1998.

40. ROMALDINI, C. C. et al. Risk factors for atherosclerosis in children and adolescents with family history of premature coronary artery disease. Jornal de Pediatria, v. 80, n. 2, p. 135-40, 2004.
41. SANTOS, D. R. III Diretrizes Brasileiras sobre Dislipidemias e Diretrizes de Prevenção da Aterosclerose da Sociedade Brasileira de Cardiologia. Arq Bras Cardiol, v. 77, supl. 3, p. 1-48, 2001.

42. SEKI, M. et al. Avaliação dos intervalos de referência de lípides e lipoproteínas para crianças e adolescentes: associação das dislipidemias e sobrepeso em escolares de 4 a 19 anos de idade da cidade de Maracaí (São Paulo). J Brasil Patol Med Lab, v. 42, n. 4, p. 265-70, 2006.

43. SEKI, M. et al. Estudo do perfil lipídico de crianças e jovens até 19 anos de idade. J Brasil Patol Med Lab, v. 37, n. 4, p. 247-51, 2001.

44. SILVA, R. A. et al. Estudo do perfil lipídico em crianças e jovens do ambulatório pediátrico do Hospital Universitário Antônio Pedro associado ao risco de dislipidemias. J Brasil Patol Med Lab, v. 43, n. 2, p. 95-101, 2007.

45. SOAR, C.; VASCONCELOS, F. A. G.; ASSIS, M. A. A. $A$ relação cintura quadril e o perímetro da cintura associados ao índice de massa corporal em estudo com escolares. Cad Saúde Pública, v. 20, n. 6, p. 1609-16, 2004.

46. SRINIVASAN, S. R.; BAO, W.; BERENSON, G. S. Coexistence of increased levels of adiposity, insulin, and blood pressure in a young adult cohort with elevated very low-density lipoprotein cholesterol: the Bogalusa Heart Study. Metabolism, v. 45, p. 170-6, 1993.

47. TERRES, N. G. et al. Prevalência e fatores associados ao sobrepeso e à obesidade em adolescentes. Rev Saúde Pública, v. 40, n. 4, p. 627-33, 2006.

48. VISCHER, T. L.; SEIDELL, J. C. The public health impact of obesity. Annu Rev Public Health, v. 22, p. 355-75, 2001.

49. WANG, Y.; MONTEIRO, C. A.; POPKIN, B. M. Trends of obesity and underweight in older children and adolescents in the United States, Brazil, China and Russia. Am J Clin Nutr, v. 75, n. 6, p. 971-7, 2002.

50. WORLD HEALTH ORGANIZATION. Obesity: preventing and managing the global epidemic. Geneva: WHO, 1997.

51. WORLD HEALTH ORGANIZATION. Physical status: the use and interpretation of anthropometry. Geneva: WHO, 1995.

\begin{tabular}{l|l} 
Endereço para correspondência \\
\hline Miguel Machinski Junior \\
Universidade Estadual de Maringá \\
Departamento de Ciências Básicas da Saúde \\
Av. Colombo, 5790 \\
CEP: 87020-900 - Maringá-PR \\
Tel./Fax: (44) 3011-4489 \\
e-mail: mmjunior@uem.br
\end{tabular}

